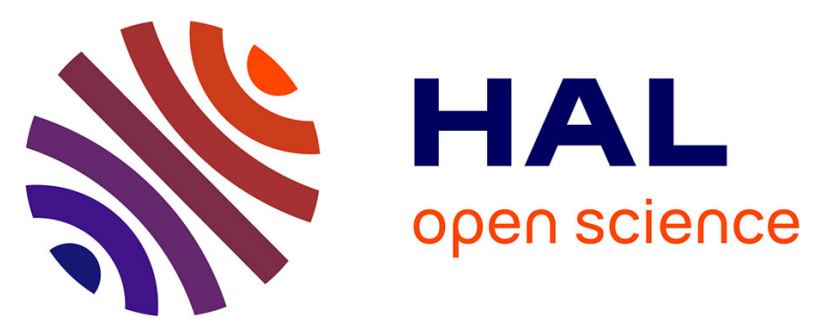

\title{
Single-sided radio-frequency field gradient with two unsymmetrical loops: Applications to nuclear magnetic resonance
}

Laouès Guendouz, Sébastien Leclerc, Alain Retournard, Ahcène Hedjiedj, Daniel Canet

\section{To cite this version:}

Laouès Guendouz, Sébastien Leclerc, Alain Retournard, Ahcène Hedjiedj, Daniel Canet. Singlesided radio-frequency field gradient with two unsymmetrical loops: Applications to nuclear magnetic resonance. Review of Scientific Instruments, 2008, 79, pp.123704 - 123704. 10.1063/1.3042276 . hal01482107

\section{HAL Id: hal-01482107 \\ https://hal.univ-lorraine.fr/hal-01482107}

Submitted on 3 Mar 2017

HAL is a multi-disciplinary open access archive for the deposit and dissemination of scientific research documents, whether they are published or not. The documents may come from teaching and research institutions in France or abroad, or from public or private research centers.
L'archive ouverte pluridisciplinaire HAL, est destinée au dépôt et à la diffusion de documents scientifiques de niveau recherche, publiés ou non, émanant des établissements d'enseignement et de recherche français ou étrangers, des laboratoires publics ou privés. 


\title{
Single-sided radio-frequency field gradient with two unsymmetrical loops. Applications
}

\section{to Nuclear Magnetic Resonance}

Laouès Guendouz ${ }^{1}$, Sébastien Leclerc ${ }^{2}$, Alain Retournard ${ }^{3}$, Ahcène Hedjiedj $^{1}$, Daniel Canet $^{3}$

${ }^{1}$ Laboratoire d'Instrumentation Electronique de Nancy, Nancy Université, Faculté des sciences et techniques, BP 239, 54506 Vandoeuvre-lès-Nancy Cedex, France

${ }^{2}$ LEMTA (UMR 7563 CNRS-Nancy Université), 2 avenue de la forêt de haye, BP 160, 54504 Vandoeuvre-lès-Nancy Cedex, France

3 Méthodologie RMN (UMR 7565 CNRS-Nancy Université), Faculté des sciences et techniques, BP 239, 54506 Vandoeuvre-lès-Nancy Cedex, France

\begin{abstract}
Magnetic field gradients are nowadays indispensable to most nuclear magnetic resonance experiments and are at the basis of magnetic resonance imaging (MRI). Most of time, gradients of the static magnetic field are employed. Gradients of the radio-frequency (rf) field may constitute an interesting alternative. Until now, they were produced by a single loop. We demonstrate in this paper how two unsymmetrical series loops can be optimized to produce rf gradients of much better performances. This optimization is based on a thorough theoretical approach and the gradient uniformity is studied through accurate simulations. Two prototypes were devised: one for a $2.34 \mathrm{~T}$ horizontal magnet (used in MRI), the other for a $4.7 \mathrm{~T}$ vertical magnet (used for pure spectroscopic applications). These two-loop systems were designed for proton resonance frequencies (100 MHz and $200 \mathrm{MHz}$, respectively). Performances of both
\end{abstract}


systems were verified (vs. theoretical predictions) by means of experiments employing gradients in view of the determination of the self-diffusion coefficients of liquids. 


\section{INTRODUCTION}

Most Nuclear Magnetic Resonance (NMR) experiments necessarily involve nowadays the use of magnetic field gradients. These experiments include i) coherence pathway selection in pure spectroscopy, ii) measurements of self-diffusion coefficients, and of course iii) Magnetic Resonance Imaging (MRI) or NMR Microscopy ${ }^{1}$. All these techniques rest generally on gradients of the static magnetic field ( $B_{0}$ gradients) for which enormous efforts of development have been carried out in the last three decades. Nevertheless, they are still hampered by the issue of the so-called internal gradients ${ }^{2}$ which superpose to the applied gradients and can therefore alter the spectroscopic or imaging data. These internal gradients arise from the magnetic susceptibility differences of the materials constituting the sample or the object under investigation. They occur at the interfaces between these materials and are especially detrimental in heterogeneous samples. Of course, many schemes have been devised for circumventing this drawback including the use of stronger and stronger applied gradients. This latter remedy is however hampered by the inevitable increase of rise and fall times of $B_{0}$ gradients, generally applied in the form of short pulses. On the other hand, gradients of the radio-frequency (rf) magnetic field ( $B_{1}$ gradients), the alternating magnetic field mandatory in any NMR experiment, have received much less attention ${ }^{3}$. They prove however to be totally immune to internal (or background) gradients since the latter are $B_{0}$ gradients in nature (of course, internal $B_{1}$ gradients exist but are totally negligible due to the amplitude of $B_{1}$ fields as compared to that of $B_{0}$ fields). On the other hand, rise and fall times are very small in the case of $B_{1}$ gradient pulses (i.e., oscillations at the beginning and at the end of the RF pulses never exceed a few hundreds of ns). All these features should render $B_{1}$ gradients rather attractive and, indeed, some phenomena such as diffusive-diffractive peaks could be observed with $B_{1}$ gradients in real porous media while they are totally missed with $B_{0}$ gradients ${ }^{4}$. Likewise, the variation of the apparent self-diffusion coefficient as a function of the diffusion interval could 
be properly observed in these systems with $B_{1}$ gradients while this variation was underestimated with $B_{0}$ gradients. In the same order of idea, NMR images can be devoid of blurring effects in heterogeneous objects when produced by $B_{1}$ gradients. These properties should justify efforts for improving the quality of $B_{1}$ gradients which suffer from some drawbacks related to their strengths or to their uniformity in an acceptable spatial zone. In the past, our group has been utilizing a single loop for producing $B_{1}$ gradients with acceptable strength and uniformity ${ }^{3}$. Other groups have sacrificed uniformity for reaching stronger gradients either with toroidal cavities ${ }^{5}$ or especially shaped solenoids possibly combined with $B_{0}$ gradients $^{6}$. A third approach makes use of two orthogonal loops for creating $B_{1}$ gradients in two directions ${ }^{7}$. Alternatively, a pair of anti-Helmholtz coils can generate an rf gradient, and this possibility with two different currents in each loops has been already used in some way to achieve spatial localization (the so-called "straddle coil",8,9), but it involves a zero magnetic field near the center of the arrangement, and does not generate an uniform gradient. Therefore, this methodology cannot be used for our applications.

It is the aim of this paper to propose a coil arrangement for circumventing some of the drawbacks mentioned above. From previous work dealing with double Helmholtz coils devised for improving the $B_{1}$ field homogeneity ${ }^{10}$, it appears that half of this arrangement is capable of generating stronger and more uniform $B_{1}$ gradients. Such arrangements will be discussed here and optimized. Tests will be carried out with dedicated probes (antennas) adapted to horizontal magnets (devoted to IRM) and to vertical magnets (devoted to high resolution NMR spectroscopy). 


\section{THEORY}

The expression of the magnetic field produced by a single loop of radius $a$ and along the symmetry axis of this loop ( $x$ in the following) is given by the well known formula derived from the Biot and Savart law.

$$
B_{1}^{\text {axial }}=\frac{\mu_{0} I}{2} \frac{a^{2}}{\left[a^{2}+(x-a / 2)^{2}\right]^{3 / 2}}
$$

$\mu_{0}$ is the vacuum permittivity and $I$ the intensity of the electric current in the loop. Note that the origin of the $x$-axis has been chosen at the location $a / 2$ where the first derivative $\left(\frac{d B_{1}}{d x}\right.$; in other words the $B_{1}$ gradient in the $x$ direction) is maximum while the second order derivative is zero (inflexion of the curve representing the $B_{1}$ variation as a function of $x$ ). Note also that this expression is strictly valid in the dc case but constitutes an excellent approximation (quasi-static approximation) for NMR experiments carried out at relatively low frequencies (up to $200 \mathrm{MHz}$ with coil dimensions significantly smaller than the wavelength). The choice of the origin of the $x$-axis makes straightforward an expansion of Eq. (1) as a function of the reduced variable $(x / a)$.

$$
B_{1}^{\text {axial }}=\frac{\mu_{0} I}{a}\left[0.357771+0.429325(x / a)-0.457947(x / a)^{3}+\ldots\right]
$$

As indicated above, the second order term vanishes but the amplitude of the third order term is slightly larger than that of the first order term (the $B_{1}$ gradient). This explains why the zone where the $B_{1}$ gradient is uniform ( $\pm 1 \%$ of its maximum value) is rather small : [-0.06 a, 0.06 a]. This latter interval is deduced, to a first approximation, from Eq. (2).

As shown before ${ }^{10}$, additional loops of dimension and location to be optimized can lead to the cancellation of higher order terms in an expansion such as (2). Our aim will be primarily the suppression of the third order term. Let us consider an arrangement made of two coaxial loops and an arbitrary origin $\mathrm{O}$ which would advantageously correspond to the inflexion point of the 
$B_{1}$ curve (see above for the case of a single loop). Owing to the axial symmetry of such an arrangement, it would be convenient to have recourse to spherical coordinates. Indeed, Roméo and Hoult ${ }^{11}$ have proposed a very useful expression which provides the axial component of the $B_{1}$ field at a location $\mathbf{M}$ specified by the vector $\mathbf{r}=\mathbf{O M}$, this vector being defined by its length $r$ and its angle $\theta$ with the $x$-axis. The Roméo and Hoult formula is expressed as follows for a single loop $i$ of radius $a_{i}$ with a current intensity $I_{i}$, its position being defined by the vector $\mathbf{R}_{\mathbf{i}}$ (with length $R_{i}$ ) joining the origin $\mathrm{O}$ to any point of the loop and by the angle $\alpha_{i}$ between $\mathbf{R}_{\mathbf{i}}$ and the $x$-axis [Fig. 1(a)].

$$
B_{1}^{\text {axial }}=\frac{\mu_{0} I_{i}}{2 R_{i}}\left\{\sum_{n=0}^{\infty}\left[\left(P_{n}\left(\cos \alpha_{i}\right)-\cos \alpha_{i} P_{n+1}\left(\cos \alpha_{i}\right)\right)(n+1)\left(\frac{r}{R_{i}}\right)^{n} P_{n}(\cos \theta)\right]\right\}
$$

where $P_{n}$ is a Legendre polynomial. For two loops with the same current throughout ( $\left.I=I_{1}=I_{2}\right)$, and limiting the calculations to the $x$ axis $\left(\theta=0 ; r=x\right.$ and $\left.P_{n}(\cos \theta)=1\right)$, we obtain (simply by superposition)

$$
B_{1}^{\text {axial }}=\frac{\mu_{0} I}{2}\left\{\sum_{i=1}^{2} \frac{1}{R_{i}} \sum_{n=0}^{\infty}\left[\left(P_{n}\left(\cos \alpha_{i}\right)-\cos \alpha_{i} P_{n+1}\left(\cos \alpha_{i}\right)\right)(n+1)\left(\frac{x}{R_{i}}\right)^{n}\right]\right\}
$$

The coefficients of the second, third and fourth order terms [in Eq. (4)] are given below as a function of $X_{i}=\cos \alpha_{i}$ 
Second order : $\frac{3 \mu_{0} I}{4} \frac{\left(-1+6 X_{i}^{2}-5 X_{i}^{4}\right)}{R_{i}^{3}}$

Third order : $\frac{5 \mu_{0} I}{4} \frac{X_{i}\left(-3+10 X_{i}^{2}-7 X_{i}^{4}\right)}{R_{i}^{4}}$

Fourth order : $\frac{15 \mu_{0} I}{16} \frac{\left(1-15 X_{i}^{2}+35 X_{i}^{4}-21 X_{i}^{6}\right)}{R_{i}^{5}}$

For the sake of simplicity, we shall limit the discussion about gradient uniformity to the cancellation of second order and third order terms. Appropriate values of $X_{1}$ and $X_{2}$ are the roots of the two following equations

$$
\begin{aligned}
& R_{21}^{3}\left(1-6 X_{1}^{2}+5 X_{1}^{4}\right)+\left(1-6 X_{2}^{2}+5 X_{2}^{4}\right)=0 \\
& R_{21}^{4} X_{1}\left(3-10 X_{1}^{2}+7 X_{1}^{4}\right)+X_{2}\left(3-10 X_{2}^{2}+7 X_{2}^{4}\right)=0
\end{aligned}
$$

where the ratio $R_{21}=R_{2} / R_{1}$ has been introduced. With the conventions of Fig. 1, one has $R_{21}>0,0 \leq X_{i}<1$ (or equivalently $0<\alpha_{i} \leq \pi / 2$ ). From a general point of view, we can decide to consider only the case $R_{21} \geq 1$, because, for the inverse ratio $R_{21} \leq 1$, the two roots $\left(X_{1}, X_{2}\right)$ become $\left(X_{2}, X_{1}\right)$. On the other hand, possible roots can be separated into two classes: one with $X_{1}$ and $X_{2}$ of identical signs, the other with $X_{1}$ and $X_{2}$ of opposite signs. Indeed, much stronger and more uniform gradients are predicted for $X_{1}$ and $X_{2}$ of identical signs, hence the choice of the single sided configuration as detailed below.

\section{Two coaxial loops on a spherical surface}

Choosing the origin at the sphere center, one has $R_{21}=1$ [Fig. 1(b)]. Although this arrangement yielded a very good result in terms of $B_{1}$ homogeneity when dealing with a pair of such a two-loop system ${ }^{10}$, it can be shown numerically that here (with the goal of improving the homogeneity of the $B_{1}$ gradient), no physically meaningful roots [to Eqs. $(8 \mathrm{a}, \mathrm{b})]$ exist. However, it is always possible to cancel the second order term [Eq. (8a)] while 
minimizing the third order term [Eq. (8b)]. We find the set $X_{1}=0.827276$ and $X_{2}=0.201604$ or, equivalently, $\alpha_{1} \approx 34.2^{\circ}$ and $\alpha_{2} \approx 78.4^{\circ}$. By reference to the single loop system, dubbed as $S L_{a}$, we shall define $a$ as $a=a_{2}=R_{2} \sin \alpha_{2}$ and dub the present two-loop system (with the above values of $X_{1}$ and $X_{2}$ ) as $S_{a}$. With these notations, and mimicking Eq. (2), we obtain

$$
B_{1}^{a x i a l}=\frac{\mu_{0} I}{a}\left[0.624396+0.654055(x / a)-0.066324(x / a)^{3}+0.712216(x / a)^{4}+\ldots\right]
$$

Compared to Eq. (2), an increase of the second term (the $B_{1}$ gradient) and a significant decrease of the third term (thus a significant improvement of the gradient homogeneity) can be observed. From Eq. (9), the $B_{1}$ field at the origin is $7.8463910^{-7} \mathrm{I} / \mathrm{a}$ T (in Tesla units) and the $B_{1}$ gradient is equal to $8.2191010^{-7} \mathrm{I} / \mathrm{a}^{2} \mathrm{~T} / \mathrm{m}$.

\section{Two coaxial loops on an ellipsoidal surface}

We consider the non-spherical case with $R_{21}>1$ [Fig. 1(c)] for which two sets of physically acceptable roots can be found in the interval $1.01448 \leq R_{21} \leq 1.15260$. One set is especially interesting and corresponds to two loops on a half-ellipsoidal surface oriented toward the origin. The particular value $R_{21}=1.123112$ is rather attractive because it leads to an arrangement that maximizes the gradient and even cancels the fourth order term (see expression. (7)) thus extending significantly the zone of gradient uniformity. For this value of $R_{21}$, we obtain $X_{1}=0.676691$ and $X_{2}=0.040172$ or, equivalently, $\alpha_{1} \approx 47.4^{\circ}$ and $\alpha_{2} \approx 87.7^{\circ}$. Still with the same convention as before $\left(a=a_{2}=R_{2} \sin \alpha_{2}\right)$, the expression of $B_{1}^{a x i a l}$ becomes (the corresponding arrangement will be denoted as $E_{a}$ in the following) 


$$
B_{1}^{a x i a l}=\frac{\mu_{0} I}{a}\left[0.802958+0.753007(x / a)-1.48861(x / a)^{5}+\ldots\right]
$$

with a $B_{1}$ field at the origin of $1.0090310^{-6} \mathrm{I} / \mathrm{a} \mathrm{T}$ and a $B_{1}$ gradient of $9.4625610^{-7}$ $I / a^{2} \mathrm{~T} / \mathrm{m}$. In addition to the considerable improvement of gradient uniformity, a significant increase of the gradient itself can be noted. Finally, beyond $R_{21}=1.15260$, only one set of physically acceptable roots can be found; it is however without interest as far as the gradient and its uniformity are concerned.

\section{Comparison of the three arrangements}

Referring to $S L_{a}$, for a given current $I$ in all coils, the $B_{1}$ value for the $S_{a}$ and $E_{a}$ arrangements increases by $74.5 \%$ and $124.4 \%$, respectively. Likewise, and more importantly in the present context, the rf field gradient increases by $52.3 \%$ and $75.4 \%$, respectively. Useful dimensions of the $S L_{a}, S_{a}$ and $E_{a}$ arrangements are given in Table I.

\section{SIMULATIONS}

\section{$B_{1}$ field and $B_{1}$ gradient along the symmetry axis}

Calculations are performed according to Eq. (1) and by adding the contributions of the two loops. $B_{1}$ profiles are shown in Fig. 2 for the three arrangements $S L_{a}, S_{a}$ and $E_{a}$ assumed to carry the same current $I$. Data are normalized with respect to the $B_{1}$ value at the origin chosen for the reference loop $S L_{a}$. As expected, the linearity zone is larger for $S_{a}$ than for $S L_{a}$ and still larger for $E_{a}$ than for $S_{a} . B_{1}$ gradient profiles are shown in Fig. 3. They have been obtained by differentiating (numerically) $B_{1}$ with respect to the variable $x$. The vertical scale of Fig. 3 has been normalized, for each arrangement, with respect to the gradient value at the origin $\mathrm{O}$. The improvement of the gradient uniformity is thus confirmed for a two-loop arrangement, especially for the arrangement $E_{a}$. The gradient homogeneity can be quantified by the width of the region corresponding to a $\pm 1 \%$ variation. These widths are [-0.0580 a, $0.0542 \mathrm{a}]$, [- 
0.1175 a, 0.1479 a], [-0.2011 a, 0.1659 a] for $S L_{a}, S_{a}, E_{a}$, respectively. Even though the latter two regions are not centered on the origin $\mathrm{O}$, it appears that the uniformity zone has more than doubled when going from $S L_{a}$ to $S_{a}$ and more than tripled when going from $S L_{a}$ to $E_{a}$. With $10 \%$ in place of $1 \%$, we obtain for the new intervals: [-0.2030 a, 0.1628 a], [-0.3107 a, 0.2859 a], [-0.4170 a, $0.2863 \mathrm{a}]$. The improvement associated with the use of two loops is seen to be here less significant.

\section{$B_{1}$ field and $B_{1}$ gradient off the symmetry axis}

From the expression (in cylindrical coordinates) given by Smythe ${ }^{12}$ for the components of the field produced by a single loop, we were able to calculate the field components in the case of two loops with the help of the Mathematica software $^{13}$. Results are displayed in Fig. 4. Let us recall that axial symmetry prevails here, $x$ denoting the symmetry axis (as above) and $y$ one axis in the transverse plane. The axial component of $B_{1}\left(B_{1 x}\right)$ is seen to be rather uniform in the transverse plane, over a zone roughly equal to the diameter of the main loop [Fig. 4(a)]. By contrast, the transverse component $\left(B_{1 y}\right)$ is far from being uniform in the transverse plane and even exhibits an almost uniform slope [Fig. 4(b)]. In fact, it is zero on the symmetry axis and changes sign when it crosses the symmetry axis. Although its value is smaller than that of the axial component, it could be a problem since this transverse rf field gradient adds to the axial rf field gradient (the main gradient). However, the transverse rf field component is zero at the sample center, consequently unable to excite the spins in the major part of the sample. Moreover, as this component has opposite signs on both sides of the sample symmetry axis, its possible effects should cancel on an average. These latter considerations lead to the conclusion that we have to consider essentially the gradient of the axial component. As a matter of fact, one of the objectives of this work is precisely to obtain the best uniformity for the axial gradient. The contour plots of Fig. 5 show clearly the size of the uniformity zone for 
the three arrangements considered here. It can be noticed that this homogeneity zone is (surprisingly) larger in the transverse direction than in the axial direction. The dimensions of the object under investigation is primarily dictated by the gradient uniformity along the axial (x) direction. The above considerations lead to the conclusion that dimensions along the other two dimensions should be similar.

\section{ELECTRICAL CIRCUIT MODEL (TOTAL EQUIVALENT INDUCTANCE)}

In order to optimize the performances of an rf antenna, it is necessary to calculate the values of the tuning and matching capacitors which implies to establish an electrical model of the antenna. Owing to the frequencies considered here, the dimensions of the device are small with respect of the wavelength so that the problem amounts to treat two series loops magnetically coupled and to determine their global inductance.

Working at high frequencies (which implies negligible skin depth), the specific inductance of a circular loop $i$ of radius $a_{i}$ with a conductor of radius $\rho_{i}$ can be approximated as ${ }^{14}$

$$
L_{i} \approx \mu_{0} a_{i}\left[\ln \left(8 \frac{a_{i}}{\rho_{i}}\right)-2\right]
$$

Likewise, the mutual inductance of two coaxial loops of radii $a_{1}$ and $a_{2}$ separated by $d$ can be written as ${ }^{12}$

$$
M_{12}=\mu_{0} \sqrt{a_{1} a_{2}} k\left[\left(\frac{2}{k^{2}}-1\right) K(k)-\frac{2}{k^{2}} E(k)\right]
$$

where $k^{2}=4 a_{1} a_{2} /\left[\left(a_{1}+a_{2}\right)^{2}+d^{2}\right], K$ and $E$ being the complete elliptic integrals of the first and second kind. Eq. (12) can be expressed more simply ${ }^{14}$

$$
M_{12}=\mu_{0} \sqrt{a_{1} a_{2}} m_{12}
$$

The quantity $m_{12}$ is dimensionless and depends solely on the geometry of the two-loop system. Relevant numerical values are reported in Table II. From these values, we can express 
the global inductance $L=L_{1}+L_{2}+2 M_{12}$ for our two arrangements $S_{a}$ and $E_{a}$ (the two loops in a serial configuration)

$$
\begin{aligned}
& L_{\left(S_{a}\right)}=\mu_{0} a\left[0.389639+1.57357 \ln \left(\frac{a}{\rho}\right)\right] \\
& L_{\left(E_{a}\right)}=\mu_{0} a\left[0.697573+1.65609 \ln \left(\frac{a}{\rho}\right)\right]
\end{aligned}
$$

The interest of these calculations lie, among other things, in a first evaluation of the tuning capacitor $C_{A}$, in parallel with the two loops in a serial configuration

$$
C_{A}=1 /\left(L \omega_{0}^{2}\right)
$$

where $\omega_{0}$ is the NMR measurement frequency expressed in $\mathrm{rad} \mathrm{s}^{-1}$. Of course, this value has to be slightly modified according to i) the impedance matching conditions, ii) the parasitic capacitors inevitably involved in the actual circuitry.

\section{MATERIAL AND METHODS}

\section{Gradient coil prototypes}

Owing to the available space and to the actual location of the sample (in the zone of gradient uniformity), our best two-loop arrangement $\left(E_{a}\right)$ could be easily tested with a horizontal superconducting magnet with a usable aperture of $20 \mathrm{~cm}$ diameter (Bruker Biospec miniimager 2.34 T, operating at the proton NMR frequency of $100.3 \mathrm{MHz}$ ). The main loop radius $a$ is equal to $1.3 \mathrm{~cm}$. The gradient coil assembly is complemented by two Helmholtz-type coils, perpendicular to the gradient coils (thus magnetically decoupled from the gradient coils). These Helmholtz coils are used for the NMR signal detection and possibly for transmitting pulses of homogeneous rf field (in the following this type of coil will be dubbed "receive coil"). All coils are machined with a copper wire of $1 \mathrm{~mm}$ diameter; they must be perpendicular to the static magnetic field $\left(B_{0}\right)$ direction. The sample is a $7 \mathrm{~mm}$ o.d. tube 
containing the substance under investigation and is placed vertically. It should be noted that the Helmholtz-type coils and, evidently, the sample are partly inside the two gradient coils so as to fully benefit from the gradient uniformity zone (see Fig. 5). Finally, for the sake of comparison, we have also built an antenna of the $S L_{a}$ type with a single gradient coil identical to the main loop of $E_{a}$ (denoted in the following by $S L_{E a}$ ).

Standard NMR spectrometers are generally equipped with a vertical superconducting magnet. The sample (generally a $5 \mathrm{~mm}$ o.d. NMR tube) is positioned vertically in the probe by means of pneumatic device which serves as well to remove the sample. This means that the sample cannot be placed inside the gradient coil system as this was the case for the $E_{a}$ configuration. For this reason, we turned to the $S_{a}$ configuration (see Fig. 5) which is however less efficient in terms of gradient strength and gradient uniformity, although it provides an enlarged gradient uniformity zone and an improved gradient strength with respect to the arrangement involving a single loop (see Figs. 2 and 3). Again, for accommodating sample tubes of $5 \mathrm{~mm}$ o.d., the main loop has a radius of $1.3 \mathrm{~cm}$ but the copper wire diameter has been slightly increased $(1.63 \mathrm{~mm})$ in order to improve the quality factor of the gradient system. Due to the necessity of having rf coils perpendicular to the static magnetic field $\left(B_{0}\right)$, the receive coil (used as well for producing homogeneous rf pulses) is of the saddle-shaped type. This is shown on the photograph of Fig. 6 (wide-bore vertical $4.7 \mathrm{~T}$ magnet: aperture of $89 \mathrm{~mm}$ ). Note that here, it is possible to install a temperature regulation device. As this was done for the $E_{a}$ arrangement, the homologous configuration with a single loop has also been tested (denoted $S L_{S a}$ in the following).

\section{Tuning and matching}

The main problem is the possible leakage between the gradient coils and the receive coil (Helmholtz-type for the horizontal magnet, saddle-shaped for the vertical magnet). In spite of 
their perpendicularity, residual couplings could remain if these coils are not part of a balanced circuit. Although an inductive coupling would be a straightforward solution ${ }^{15-16}$, due to space limitations, we had rather recourse to a capacitive coupling using a balanced tuning and matching network. The corresponding circuit is schematized in Fig. 7 and follows well known principles $^{17}$. The coil(s) is(are) assumed to possess a resistor $r$. The capacitors $C_{T}$ and $C_{M}$ contribute mainly to tuning and matching, respectively while the capacitor $C_{S}$ leads to the electrical balancing of the probe coil. It compensates, to a first approximation, half the coil impedance

$$
C_{S}=2 C_{A}=1 /\left(L \omega_{0}^{2} / 2\right)
$$

assuring that voltages are opposite in sign at both extremities of the coil assembly. In Table III, are reported the theoretical value of the global inductance $L$ for all the arrangements considered here along with the value of the different capacitors used here for the considered proton Larmor frequency and yielding a quality factor $Q$ in the range 100-300.

Capacitors must be non-magnetic and capable of handling high voltages, because of the usual high intensity of rf pulses, implying power amplifiers with an output of $300 \mathrm{~W}$ or even $1 \mathrm{~kW}$. The capacitors used in this work are of commercial origin: fixed non-magnetic capacitors (100E series, American Technical Ceramics, Huntingdon Station, NY) for adjustment and for $C_{S}, 0.8-10 \mathrm{pF}$ variable non-magnetic capacitors (RP series, Polyflon, Norwalk, CT) for fine tuning and matching.

Different tests have been performed outside the NMR magnet in view of determining the $Q$ factor and the possible influence of the liquid contained in the sample tube (load). The load does not lead to significant modifications while tests repeated inside the magnet show that the latter produces negligible effects. The $Q$ values for all the coils devised for this work are reported in Table IV. 
From a practical point of view, in spite of the electrical balancing of the gradient coil and the NMR receiving coil, it remains nevertheless a residual coupling between the coils because of their construction and positioning. However, the isolation between the two rf coils can be improved by adjusting finely their relative position (in principle, without calling into question their orthogonality). For all the tested probes, the isolation factor between the gradient coil and the receiving coil was at least $35 \mathrm{~dB}$, and we have not observed any (unwanted) rf power transmitted to the amplifiers. Overall, no particular measure (e.g., active decoupling using pin diodes) was required.

\section{NMR EXPERIMENTAL VERIFICATIONS}

An especially severe experiment for assessing the quality of an rf gradient is the measurement of a self-diffusion coefficient ${ }^{3}$. The experiment itself is very simple: it starts with a first gradient pulse (of duration $\delta$ ) which defocuses the nuclear magnetization (or rather achieves a spatial labeling), then comes an interval $\Delta$ (with $\Delta>>\delta$ ) generally called the diffusion interval and the sequence ends with a second gradient pulse, identical to the first which refocuses half of the nuclear magnetization provided that the molecules bearing the nuclear spins have not moved during $\Delta$. If diffusion occurred, the nuclear magnetization decreases so that the measured NMR signal (as obtained after a standard $90^{\circ}$ observing pulse, produced by the receive coil) is of the form

$$
S(g, \delta) \approx \exp \left(-\gamma g^{2} \delta^{2} D \Delta\right)
$$

$\gamma$ is the gyromagnetic constant of the considered nucleus, $g$ is the gradient strength and $D$ the self-diffusion coefficient. We shall assume that gradient pulses are perfectly identical, meaning that the interval $\Delta$ is sufficiently long for allowing the amplifier to fully recover. Moreover, we assume that the product $g \times \delta$ is sufficient to produce a significant decay. The experiments discussed below have been performed by varying (incrementing) $\delta$. Observing a 
decay which is not perfectly Gaussian (as implied by Eq. (18)) indicates exclusively gradient non uniformity. Of course, these discrepancies will manifest themselves primarily for important values of the product $g \times \delta$. Moreover, if the gradient is not perfectly uniform, the first gradient pulse will defocus nuclear magnetization imperfectly and if $\Delta$ is relatively short, the effect of these imperfections does not vanish by relaxation phenomena. This can be a more indirect consequence of gradient non-uniformity.

Results shown in Fig. 8 demonstrate clearly the advantages of the arrangement $E_{a}$ over a simple loop identical to the main loop of $E_{a}\left(S L_{E a}\right)$. Not only has the gradient strength increased as evidenced by a faster decay, but also the gradient uniformity is perfect for $E_{a}$ and poor for the single loop. This can be appreciated, in the $E_{a}$ case, by the coincidence of experimental data points with the theoretical curve.

The last example (Fig. 9) demonstrates the performances of the arrangement $S_{a}$ which is appropriate for a vertical NMR magnet. It is interesting to notice that the diffusion coefficient of octanol $\left(1.310^{-6} \mathrm{~cm}^{2} \mathrm{~s}^{-1} ; 20\right.$ times smaller than that of water) can be properly measured with a relatively weak gradient and a probe which has not been (for practical reasons) completely optimized (junction wires could have been shortened to minimize losses and improve the signal to noise ratio of the coil systems). Nevertheless, the good adjustment of experimental data with the theoretical curve demonstrates again the improvement of gradient uniformity with an asymmetric two-loop system. Finally, it appears that, because its sufficient size is sufficiently large with respect to the rf gradient coil dimensions, the metallic shield installed around the $S_{a}$ probe does not disturb significantly the rf gradient field.

\section{CONCLUSION}

It has been demonstrated here that, concerning gradients of the NMR rf field ( $B_{1}$ gradients), it was possible to go well beyond the performances of a single loop which, for years, seemed to 
be an acceptable compromise. This is a first attempt to use a multi-loop system in order to improve both gradient strength and gradient uniformity. In this work, we tried to obtain the best performances from a simple system of two asymmetric loops. Thanks to an appropriate theoretical approach, we were able to predict, for the more efficient arrangement, that the gradient strength is roughly twice the one which can be obtained with a single loop (for the same current intensity) while the gradient uniformity is multiplied by a factor of three (zone where the axial field gradient does not vary by more than $\pm 1 \%$ ). However, this implies a single-sided configuration with the object under investigation located inside the loop system. For the usual applications of NMR, this configuration is not suitable due to the size of standard NMR tubes and to the way they are manipulated. We were nevertheless able to devise another two-loop arrangement, still single-sided, but such that the uniformity zone can accommodate a standard NMR tube at the expense of slightly degraded performances. Adding more loops would probably lift this limitation. This issue is presently underway.

\section{ACKNOWLEGMENTS}

This work is part of the ANR project "Instrumentation in Magnetic Resonance" (Grant Blan06-2_139020). We thank J.F. Pautex for assistance in the preparation of the figures for this paper. 


\section{References}

${ }^{1}$ R. Kimmich, NMR : Tomography, Diffusometry, Relaxometry, Springer, Berlin, (1997).

${ }^{2}$ W. S. Price, Conc. Magn. Reson., 10, 197, (1998).

${ }^{3}$ D. Canet, Prog. Nucl. Mag. Reson. Spectros., 30, 101 (1997).

${ }^{4}$ J.F. Kuntz, G. Trausch, P. Palmas, P. Mutzenhardt, and D. Canet, J. Chem. Phys. 126, 134904 (2007).

${ }^{5}$ K. Woelk, J. W. Rathke, and R. J. Klingler, J. Magn. Reson. A, 105, 113 (1993).

${ }^{6}$ R. Kimmich, B. Simon, and H. Köstler, J. Magn. Reson., 112, 7 (1995).

${ }^{7}$ H. Robert and D. Pusiol., J. Magn. Reson., 127, 109 (1997).

${ }^{8}$ J.O. Friedrich and R. Freeman, J. Magn. Reson., 68, 582 (1986).

${ }^{9}$ J.O. Friedrich and R. Freeman, J. Magn. Reson., 77, 101 (1988).

${ }^{10}$ L. Guendouz, S.M.O.A. Ghaly, A. Hedjiedj, J.-M. Escanye, and D. Canet, Conc. Magn. Reson. B, 33, 9 (2008).

${ }^{11}$ F. Roméo and D.I. Hoult, Magn. Res. Med. 1, 44 (1984).

${ }^{12}$ W.R. Smythe, Static and Dynamic Electricity, $3^{\text {rd }}$ ed. (McGraw-Hill, New york, 1968).

${ }^{13}$ S. Wolfram, The Mathematica Book, $4^{\text {th }}$ ed. (University Press, Cambridge, 1999).

${ }^{14}$ F.W. Grover, Inductance calculations (Dover Publications, New York, 1946).

${ }^{15}$ M. Decorps, P. Blondet, H. Reutenauer, and J.P. Albrand, J. Magn. Reson., 65, 100 (1985).

${ }^{16}$ D.I Hoult, and B. Tomanek, Conc. Magn. Reson. B, 15, 262 (2002).

${ }^{17}$ J. Mispelter, M. Lupu, and A. Briguet, NMR Probeheads for Biophysical and Biomedical Experiments - Theoretical Principles and Practical Guidelines (Imperial College Press, London, 2006). 


\section{Tables}

TABLE I. Dimensions (relatively to the radius $a$ of the main loop) of the $S L_{a}, S_{a}$ and $E_{a}$ gradient-coils.

\begin{tabular}{llccc}
\hline & & \multicolumn{3}{c}{ Coil } \\
\cline { 3 - 4 } & & $S L_{a}$ & $S_{a}$ & $E_{a}$ \\
\hline Main loop & Radius & 1 & 1 & 1 \\
& Distance from O & 0.5 & 0.2058 & 0.0402 \\
Secondary & Radius & --- & 0.5736 & 0.6561 \\
loop & Distance from O & --- & 0.8446 & 0.6030 \\
\hline
\end{tabular}

TABLE II. Inter-loop distance $d$ (relatively to $a$, radius of the main coil) and values of the $m_{12}$ dimensionless factor in the expression of the mutual inductance $M_{12}$ (see Eqs. (12) and (13)) for the arrangements $S_{a}$ and $E_{a}$.

\begin{tabular}{ccc}
\hline & \multicolumn{2}{c}{ Coil } \\
\cline { 2 - 3 } & $S_{a}$ & $E_{a}$ \\
\hline$d / a$ & 0.6389 & 0.5628 \\
$m_{12}$ & 0.3852 & 0.5201 \\
\hline
\end{tabular}

TABLE III. Theoretical values for the gradient coil prototypes: the total inductance $L$, the capacitor $C_{A}$ for an isolated system, and the capacitors $\left(C_{S}, C_{T}, C_{M}\right)$ in the case of a capacitive coupling (see Fig. 7) and a quality factor $Q$ lying in the 100-300 range.

\begin{tabular}{lcccc}
\hline Circuit & \multicolumn{2}{c}{ Two-loop coil } & \multicolumn{2}{c}{ One-loop coil } \\
\cline { 2 - 5 } elements & $E_{a}$ & $S_{a}$ & $S L_{E a}$ & $S L_{S a}$ \\
\hline$L(\mathrm{nH})$ & 99.5 & 77.6 & 54.5 & 46.5 \\
$C_{S}=2 C_{A}(\mathrm{pF})$ & 50.7 & 16.5 & 92.5 & 27.5 \\
$C_{T}(\mathrm{pF})$ & $45.1-47.4$ & $14.2-15.2$ & $85.0-88.1$ & $24.6-25.8$ \\
$C_{M}(\mathrm{pF})$ & $5.76-3.29$ & $2.32-1.33$ & $7.90-4.47$ & $3.02-1.72$ \\
\hline
\end{tabular}

TABLE IV. Experimental values of the quality factor $Q$ of all the prototypes of the rf coils employed in this work (measured outside the magnet).

\begin{tabular}{lcccccc}
\hline & \multicolumn{2}{c}{ Two-loop coil } & \multicolumn{2}{c}{ One-loop coil } & \multicolumn{2}{c}{ Receive coil } \\
\cline { 2 - 7 } & $E_{a}$ & $S_{a}$ & $S L_{E a}$ & $S L_{S a}$ & Helmholtz-type & Saddle-type \\
\hline Load & water & octanol & water & octanol & water & octanol \\
Tube outer diameter $(\mathrm{mm})$ & 7 & 5 & 7 & 5 & 7 & 5 \\
Quality factor $Q$ & 180 & 145 & 152 & 111 & 138 & 155 \\
\hline
\end{tabular}




\section{Figure captions}

FIG. 1. (a) Notations for characterizing the location and the geometry of a single loop (SL in text). (b) Assembly of two loops on a spherical surface (S). (c) Assembly of two loops on an ellipsoidal surface $(E)$.

FIG. 2. Computed normalized $B_{1}$ axial field profiles for the coils $S L_{a}, S_{a}$ and $E_{a}$ (from bottom to top). Normalization is based on the value at the center of the reference coil $S L_{a}$. The horizontal scale represents a relative distance (with respect to the radius of the main coil).

FIG. 3. Normalized gradient profile (with respect to the value at the origin O) along the axial symmetry axis, for the three geometries $S L_{a}, S_{a}$ and $E_{a}$. Horizontal dashed lines indicate the $90 \%, 99 \%, 101 \%$ and $110 \%$ levels. The horizontal scale represents a relative distance (with respect to the radius of the main coil).

FIG. 4. rf field components for the three considered arrangements $\left(S L_{a}, S_{a}\right.$ and $\left.E_{a}\right)$ : (a) axial component $\left(B_{1 x}\right)$, (b) transverse component $\left(B_{1 y}\right)$. Normalization is based on the value at center for the reference coil $S L_{a}$. The horizontal scale represents a relative distance (with respect to the radius of the main coil).

FIG. 5. Contour plots showing the region where the axial gradient does not vary by more than $\pm 1 \%, \pm 5 \%, \pm 10 \%, \pm 15 \%, \pm 20 \%$ for the three geometries: (a) $S L_{a}$, (b) $S_{a}$ and (c) $E_{a}$. The different levels are alternately drawn with solid and dotted lines. Dots stand for the intersection of the loop wire with the considered plane which is a plane containing the $x$-axis (see Fig. 1). Note the increase of the gradient uniformity zone when going from $S L_{a}$ to $S_{a}$ and to $E_{a}$. Both axes have the same scale (relative to the radius of the main loop). 
FIG. 6. Photograph of the rf gradient coil prototype $S_{a}$ and its associated saddle-shaped coil operating in a vertical magnet at $199.01 \mathrm{MHz}$. Note that in the $100.3 \mathrm{MHz}$ coil system operating in a horizontal magnet, the gradient coil arrangement is of type $E_{a}$ and the receiving coil is a vertical Helmholtz pair.

FIG. 7. Schematic diagram of the (balanced) tuning and matching network, used systematically for all rf coils.

FIG. 8. Results of diffusion experiments obtained with $S L_{E a}$ (lozenges) and $E_{a}$ (triangles). Both antennas can be run in the horizontal magnet. The same rf power is used for both arrangements so that the $\mathrm{rf}$ gradient delivered by $S L_{E a}$ is equal to $68.5 \mathrm{mT} \mathrm{m}^{-1}$ while the gradient produced by $E_{a}$ climbs to $89.5 \mathrm{mT} \mathrm{m}^{-1}$. The sample tube $(7 \mathrm{~mm}$ o.d.) contains water. $\Delta$ is set at 0.25 s. $\delta$ values are represented on the horizontal scale. Solid lines represent the best fit to experimental data according to Eq. (18).

FIG. 9. Results of a diffusion experiment performed with the antenna $S_{a}$ (Fig. 6) in the vertical 4.7 $\mathrm{T}$ magnet. The $\mathrm{rf}$ gradient is equal to $68 \mathrm{mT} \mathrm{m}^{-1}$. A classical NMR $5 \mathrm{~mm}$ o.d. tube, filled with octanol, is set into (or removed from) pneumatically the NMR probe. $\Delta$ is set at $0.5 \mathrm{~s} . \delta$ values are represented on the horizontal scale. The solid line represents the best fit to experimental data according to Eq. (18).

Figure 9 


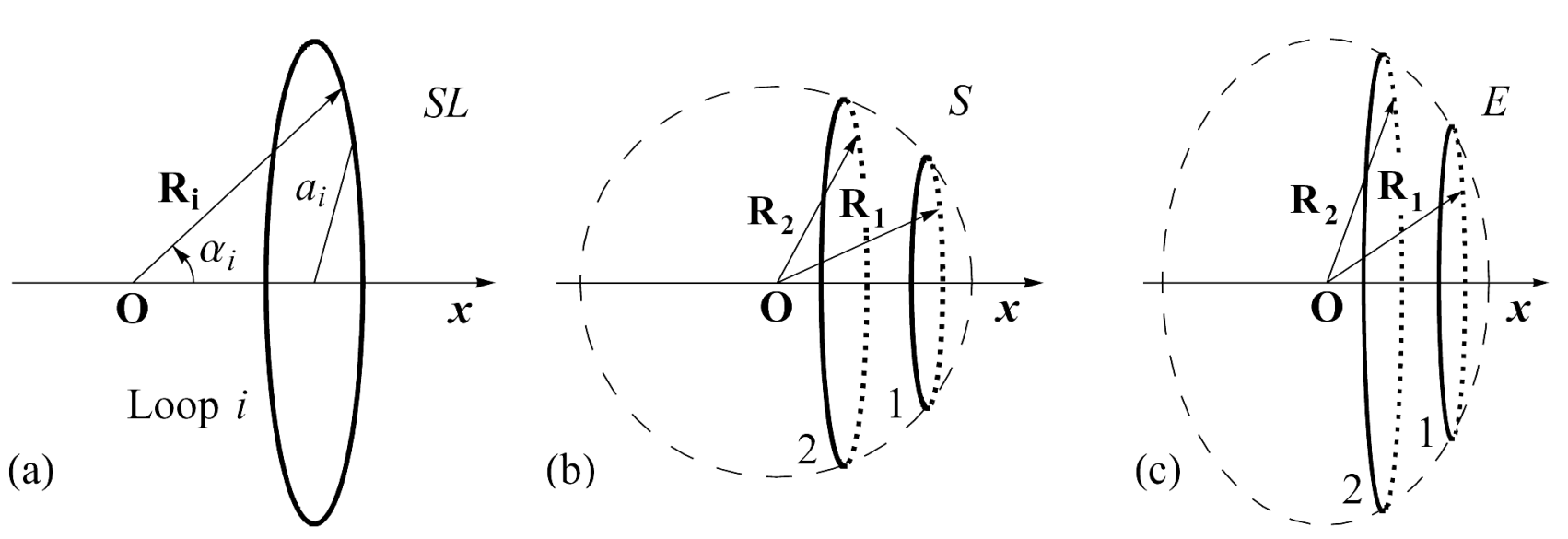




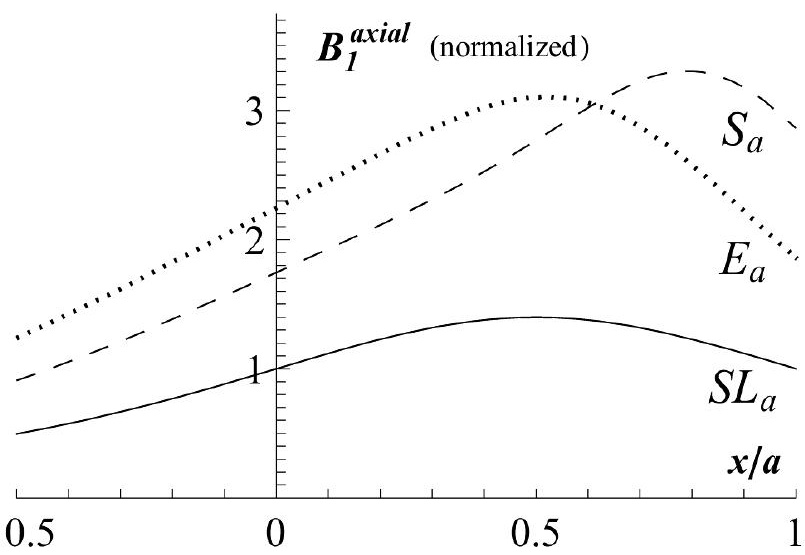




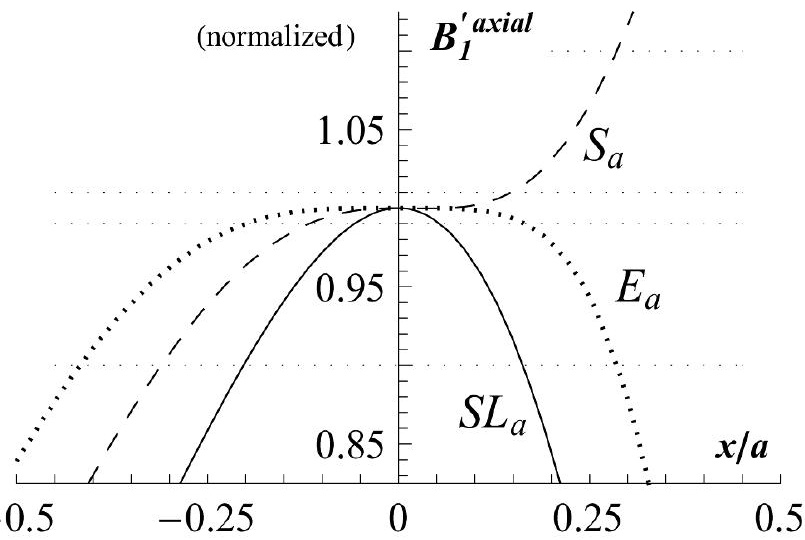



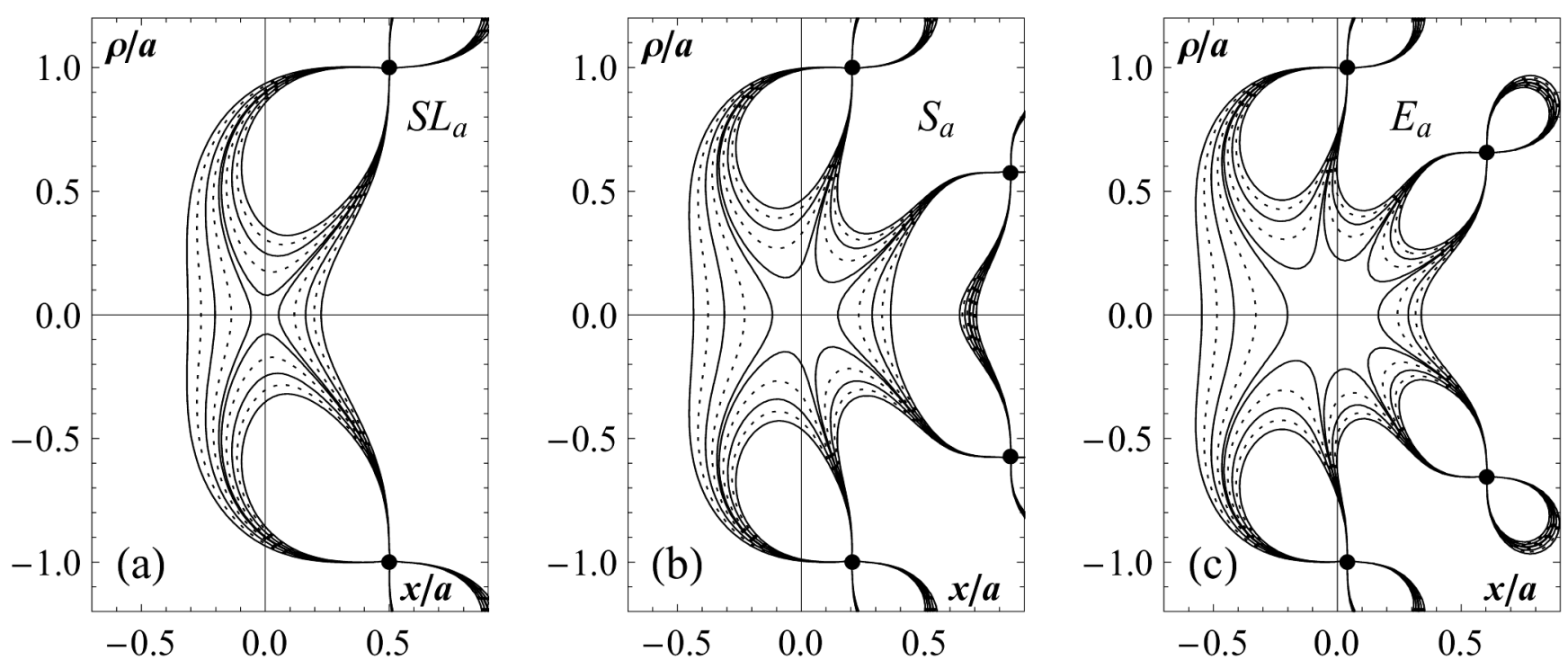


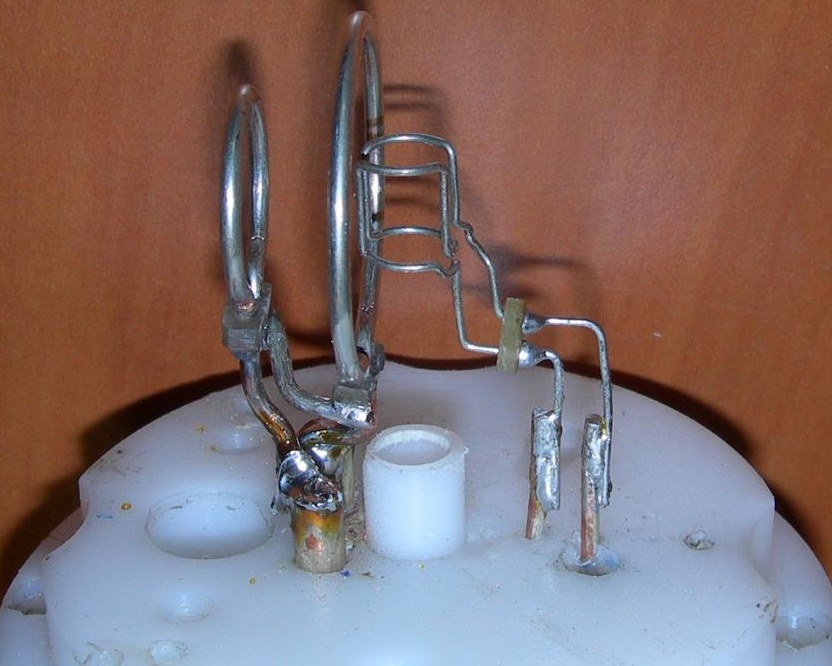




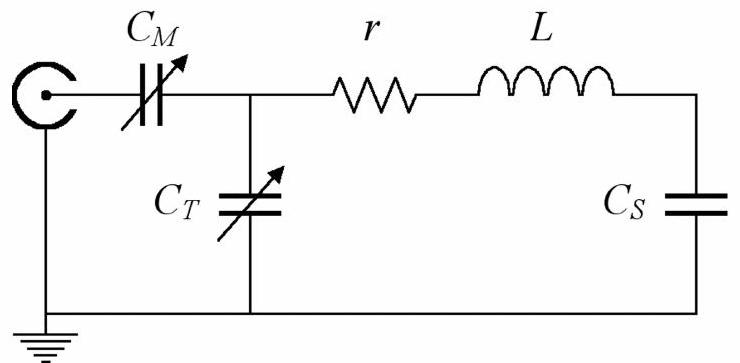




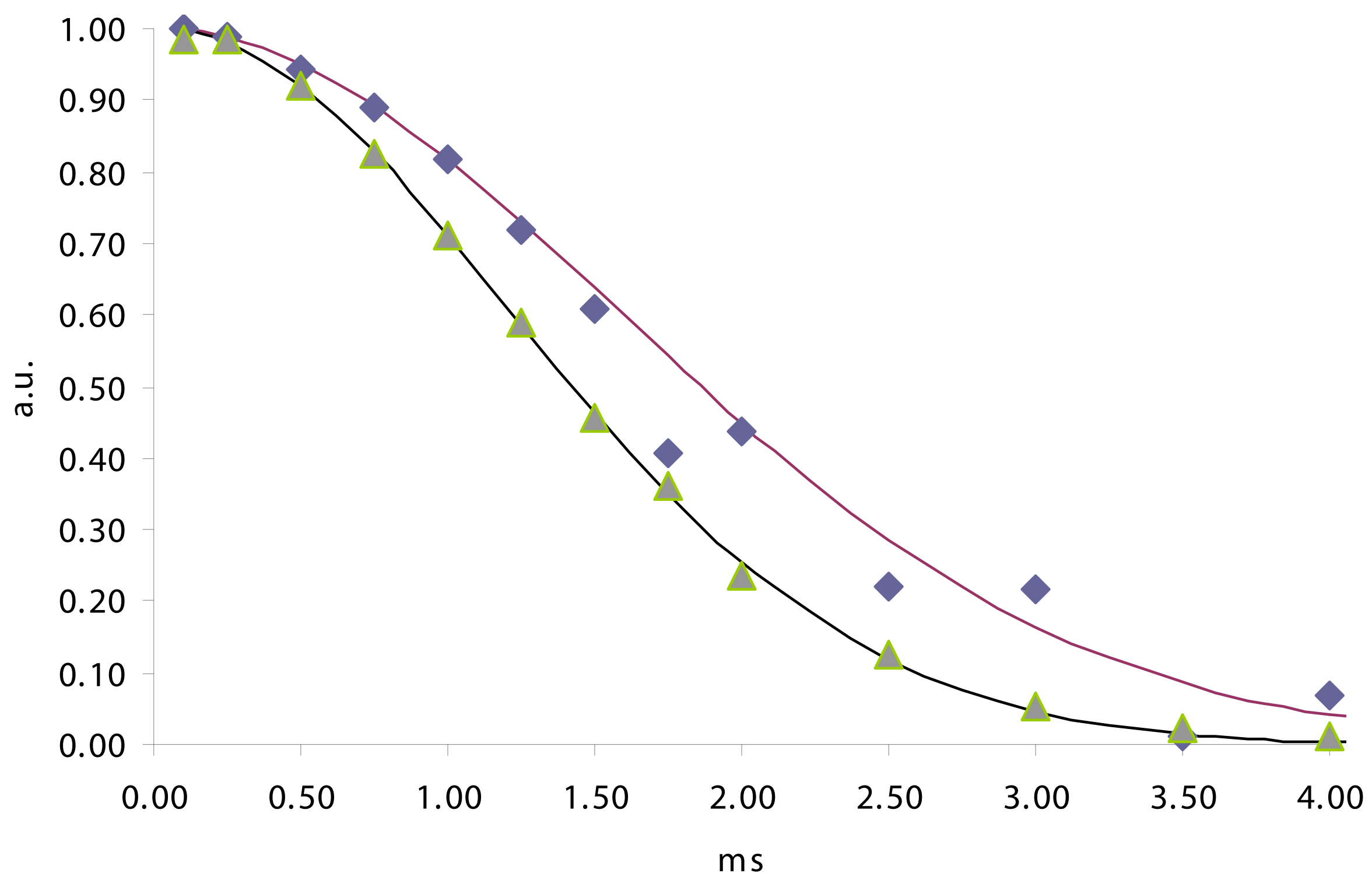




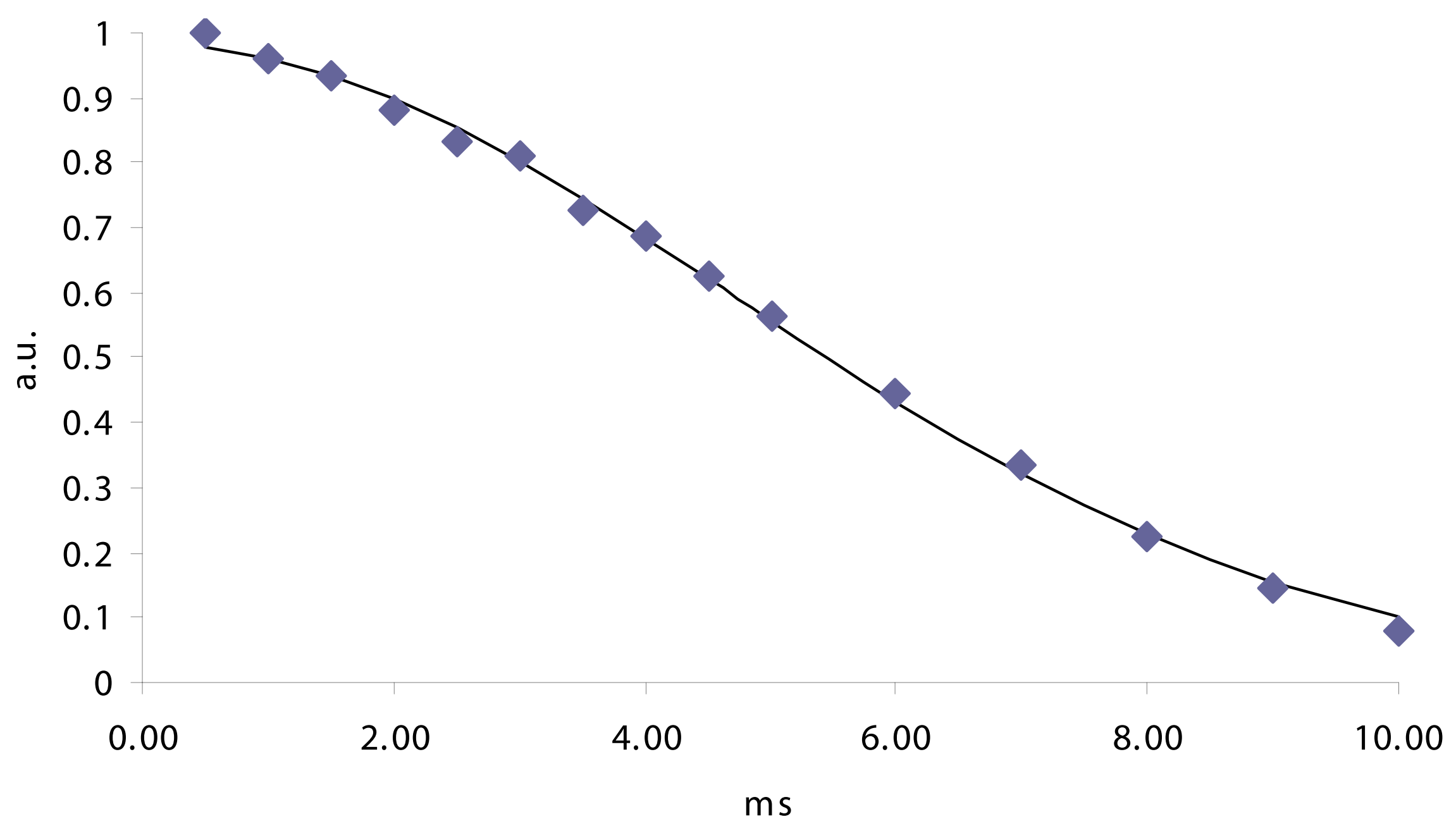

